\title{
APPLICATION OF SDMA WITH SCHEDULING FOR MIMO MC-CDMA
}

\author{
Valdemar Monteiro(1), Jonathan Rodriguez ${ }^{(1)}$, Atílio Gameiro ${ }^{(2)}$ \\ (1) Instituto de Telecomunicações, Univ. Aveiro, 3810-193, Aveiro, Portuga, Email:\{vmonteiro|jonathan\}@av.it.pt \\ (2) Dept. Electrónica \& Telecomunicações Univ. Aveiro, 3810-193, Aveiro, Portugal Email: amg@det.ua.pt
}

\begin{abstract}
Incoming Beyond $3 \mathrm{G}$ systems provides high data rates by using several techniques including Beamforming. Convenient DRA design is required to optimize the capabilities offered by smart antennas. This paper is to presents a DRA algorithm based on the HSDPA of UMTS, including scheduling that exploit SDMA technique that was implemented in the ambit of the IST 4MORE project. Results shown cell throughput gain of SDMA compared to sectorized cells about $76 \%$, and that SDMA gain will increase with user diversity within the cell.
\end{abstract}

\section{INTRODUCTION}

Demand for high bandwidth multimedia services targeting high mobility provided the impetus to supersede legacy technologies, such as UMTS and HSDPA that can currently target a peak data rate at 2 Mbps, and 12 Mbps respectively. The ITU-R (International Telecommunication Union Radio communication Sector) responsible for setting the evolutionary roadmap towards $\mathrm{B} 3 \mathrm{G}$ has defined the next milestone [1]: a beyond IMT 2000 system (International Mobile Telecommunications) that can support up to 100 Mbps peak bit rate in high mobility scenarios. The MATRICE (MC-CDMA Transmission Techniques for Integrated Broadband Cellular Systems) project was a European candidate solution for $\mathrm{B} 3 \mathrm{G}$ cellular communications that investigated the feasibility of MCCDMA to provide broadband access on-the-move in a cost-effective manner. The MATRICE vision targeted a maximum information bit rate of more than $2-20 \mathrm{Mbps}$ in a vehicular environment and possibly $50-100 \mathrm{Mbps}$ in indoor to pedestrian environments, using a $50-100 \mathrm{MHz}$ bandwidth [2]. Especially in the downlink, high throughput is needed since the number of downloads of large data files from web sites and servers will increase and broadcast / multicast services may become a reality that has to be accommodated by $4 \mathrm{G}$ systems. To support the transport of variable size IP packet over the radio, a Dynamic Resource Allocation (DRA) [3-5] scheme was designed that uses a HARQ (Hybrid Automatic Repeat ReQuest) transmission protocol based on Chase Combining, link adaptation scheme, code allocation policy and scheduling to select the appropriate transmission format according to the channel conditions and service requirements. However, to progress towards a spectrally efficient communication systems with increased cell capacity through MTMR techniques (Multiple Transmit Multiple Receiver), the 4MORE project investigated a cost effective, low power System on Chip (SoC) solution for multi-antenna MC-CDMA mobile terminals based on the joint optimization of L1 (Layer1) and L2 (Layer 2) functions [6].

In order to fully utilise the potential offered by MIMO (Multiple Input Multiple Output) signal processing techniques at $\mathrm{L} 1$, we propose a cross-layer design that uses Dynamic Resource Allocation (DRA) with SDMA (spatial division multiple access) to ensure the seamless transport of variable size IP packets over the airinterface. SDMA has been addressed extensively [7-9] as a means of tapping into the high data rates on offer by using multiple antennas. The joint application of smart antennas with MIMO has the ability to allocate selected users a common radio resource given that they can be spatially separated.

In this paper, we aim to address the system level aspect of the 4MORE air-interface in an attempt to quantify the broadband capabilities of the system thanks to the MIMO signal processing techniques introduced at L1. We propose an integrated dynamic resource allocation architecture that exploits cross-layer parameters to facilitate the allocation of users in the spatial domain in a cost effective manner whilst still ensuring Quality of Service.

This paper considers the following sections: section 2 provides an overview of the SDMA principle considered in this work; Section 3 describes the Dynamic Resource Allocation (DRA) that exploits SDMA using MIMO transmission techniques, specifically we emphasize the main design assumptions that were targeted for the simulation scenarios; in section 4 the system level simulation parameters for the targeted scenarios and simulation results are presented; and the conclusions are given in section 5 .

\section{SYSTEM MODEL WITH SDMA}

\subsection{SDMA principle}

SDMA exploits MIMO transmit techniques to increase cell capacity by facilitating the allocation of users in the spatial domain. Beamforming is based on the principle that an antenna pattern is steered by applying a weight, i.e. a complex number to each antenna element. The 
pattern weight is represented by a weight vector, which contains one weight per antenna element. The linear nature and number of antenna elements enables the end user to point the antenna beam to a selected direction, maximising the Signal-to-Interference plus Noise ratio (SINR) for the desired data stream, whilst minimising potential co-channel interference.

The application of smart antennas at the system level allows two users to be allocated the same resource as long as they can be spatially separated, the separation distance dependant on the antenna radiation pattern as shown by Fig. 1. By adjusting the antenna weights to maximise the SINR, two antenna beams can be pointing at the desired co-channel users $\mathrm{MS}_{\mathrm{i}}$, and $\mathrm{MS}_{\mathrm{j}}$, and will suffer minimal cross talk between beams as long as their angular separation (AoS) $\theta_{i, j}$ is sufficiently apart; a value dependent on the on the antenna beamwidth. This principle can be applied to both the downlink (DL) and the uplink (UL).

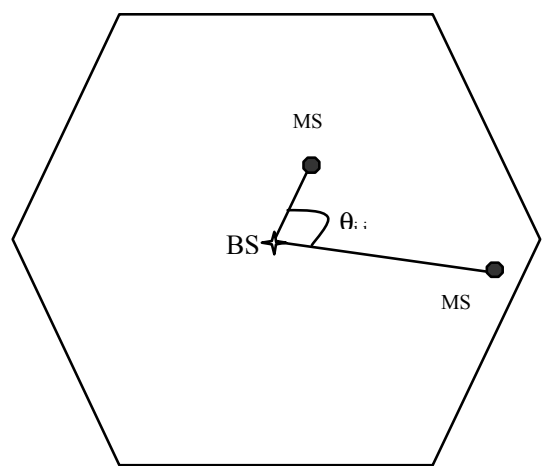

Figure 1 SDMA principle based on angular separation

\subsection{Antenna pattern}

Fig. 2 shows the antenna pattern used by the transmitter. This pattern is obtained theoretically with an array with four antennas separated by 0.5 wavelengths. This pattern consists in a $3 \mathrm{~dB}$ beamwidth of about 35 degrees and a maximum side lobe of $-13.46 \mathrm{~dB}$. Although, in the simulations we assume that no co-channel interference exists for $\theta_{i, j} \geq 45$ degrees.

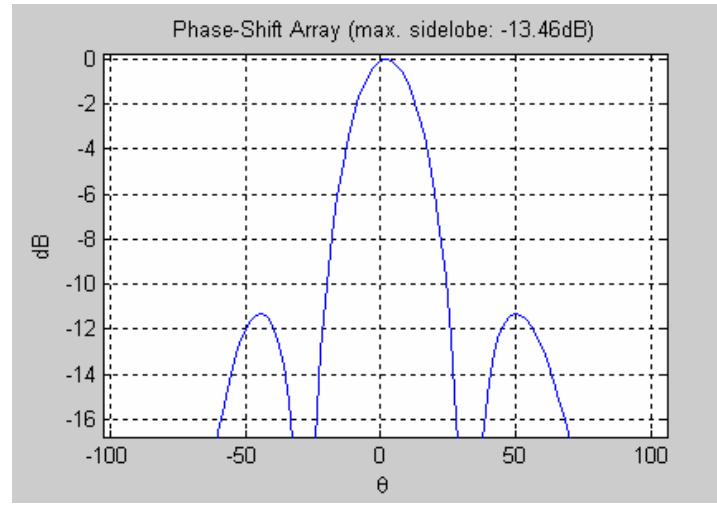

Figure 2. Smart Antenna beamforming pattern
Fig. 3 presents the antenna pattern proposed by 3GPP for a 3 -sector cell with a $3 \mathrm{~dB}$ beamwidth of 70 degrees. The gain for this antenna is $14 \mathrm{dBi}$. By reducing the beamwidth by half, to 35 degrees, the corresponding gain will be $3 \mathrm{~dB}$ higher resulting in $17 \mathrm{dBi}$ [10]. This is the corresponding antenna gain used for the SDMA simulations.

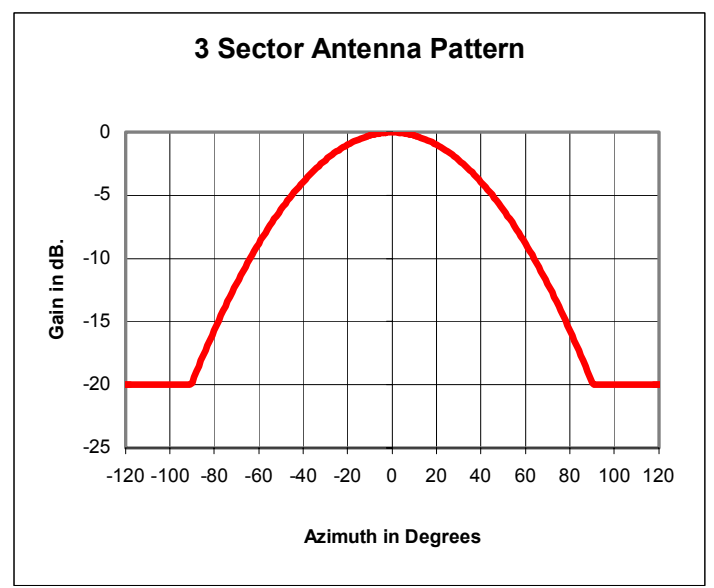

Figure 3 Antenna pattern for 3 sectors cells proposed by $3 \mathrm{GPP}$

\section{DYNAMIC RESOURCE ALLOCATION}

DRA is the entity located at the Medium Access Control (MAC) layer responsible for mapping the user logical channels to the available transport channels in a cost effective manner. DRA is designed so as to optimize the radio resource usage whilst maintaining service quality. The proposed DRA architecture targeted in 4MORE is based on [11] and [12]. It constitutes Link Adaptation, Link Layer Interface, Scheduling and Hybrid Automated Request (H-ARQ), each described in the next subsections.

\subsection{Link Adaptation}

Link adaptation is used to adjust transmission parameters due to wireless channel and offers several challenges. Adaptive Modulation and Coding (AMC) falls under a category of link adaptation by adapting the appropriate modulation and channel coding rate to the variations of the wireless channel. First of all, AMC is sensitive to channel measurement error and delay. In order to select the appropriate modulation the scheduler must be aware of the channel quality. Errors in the channel estimation will cause the scheduler to select the wrong data rate and either, in some cases transmit at too high power, wasting system capacity, or too low power, raising the block error rate. Delay in reporting channel measurements also reduces the reliability of the channel quality estimation due to the constantly varying mobile channel. Furthermore, changes in the interference add errors to the measurement. HARQ enables the implementation of AMC by reducing the number of 
required Modulation and Coding Scheme (MCS) levels and the sensitivity to measurement error and traffic fluctuations.

\subsubsection{Channel Quality Indicator}

Concerning the quality of the wireless channel, in the proposed Radio Resource Management (RRM) scheme it is assumed that the Node-B will select the transport format according to the Channel Quality Indication (CQI) reported by the user equipment (UE) [12]. It is also assumes perfect channel estimation by the UE. However there is an associated delay between the channel estimation and reporting to the Node-B. The delay between channel estimation by the UE and reporting to the Node-B is two frames. An IIR (Infinite Impulse Response) filter was used to smooth the channel estimations, with new data being weighted by $70 \%$ and the previous filter estimate being weight by $30 \%$.

\subsubsection{MCS selection algorithm}

The Modulation and Coding Scheme selection is defined by the following statement which allows a $90 \%$ probability of successful transmission at the highest MCS according the Channel Quality Indicator (CQI) in the transmission interval (TTI) $t$ :

$$
\operatorname{MCS}(t)=\underset{\arg i}{\operatorname{AaX}}(\operatorname{MCSi}((1-\operatorname{BLER}(\mathrm{CQI}(\mathrm{t}))) \geq 0.9)
$$

where the $B L E R$ is the predicted Block Error probability related to the CQI identification during the TTI $t$.

\subsection{Link Layer Interface}

The interfacing to the link layer is based on a set of look-up tables (LUT) simulated using the 4MORE air interface [13]. The mapping between SIR and BLER is based on the EESM methodology [14]. The LUT (Fig. 4) covers the transport formats based on UMTS convolutional channel coding having the constraint length $\mathrm{K}=9$ and rates equal $1 / 2,2 / 3$ and $3 / 4$ rate encoding using QPSK and 16QAM modulations, with the respective payloads presented in Tab. 1.

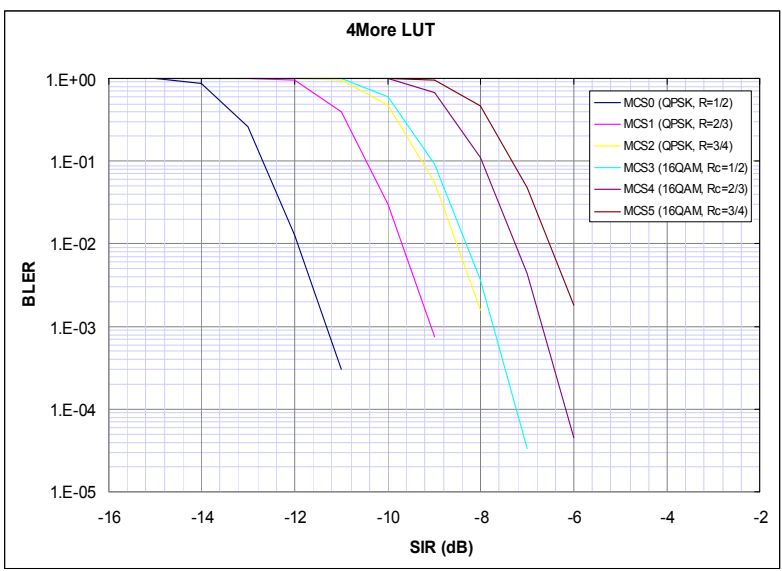

Figure 4 EESM LUT
Table 1. MCS and respective payloads

\begin{tabular}{|c|c|c|}
\hline \multicolumn{1}{c}{$\begin{array}{c}\text { Modulation and } \\
\text { Coding Rate }\end{array}$} & $\begin{array}{c}\text { Block Size } \\
\text { (bits) }\end{array}$ & $\begin{array}{c}\text { Max. } \\
\text { Bit rate } \\
\text { (Mbps) }\end{array}$ \\
\hline QPSK, R=1/2 & 504 & 15.12 \\
\hline QPSK, $R=2 / 3$ & 672 & 20.16 \\
\hline QPSK, R=3/4 & 756 & 22.68 \\
\hline 16QAM, R=1/2 & 1008 & 30.24 \\
\hline 16QAM, R=2/3 & 1344 & 40.32 \\
\hline 16QAM, R=3/4 & 1512 & 45.36 \\
\hline
\end{tabular}

\subsection{Scheduling Policy}

The DRA is based on a TDD system, with the resource space illustrated by Fig. 5. Resource space is defined in terms of time slot, code and space. The frame size is three slots, two slots allocated to DL and one slot allocated to the UL. The system evaluation however will consider only DL.

In the DL, each time slot and spatial bin consists of 32 codes, where 30 codes are allocated for data transmission, one code is allocated to the common pilot channel (CPICh) and one code is allocated to the broadcast channel $(\mathrm{BCH})$.

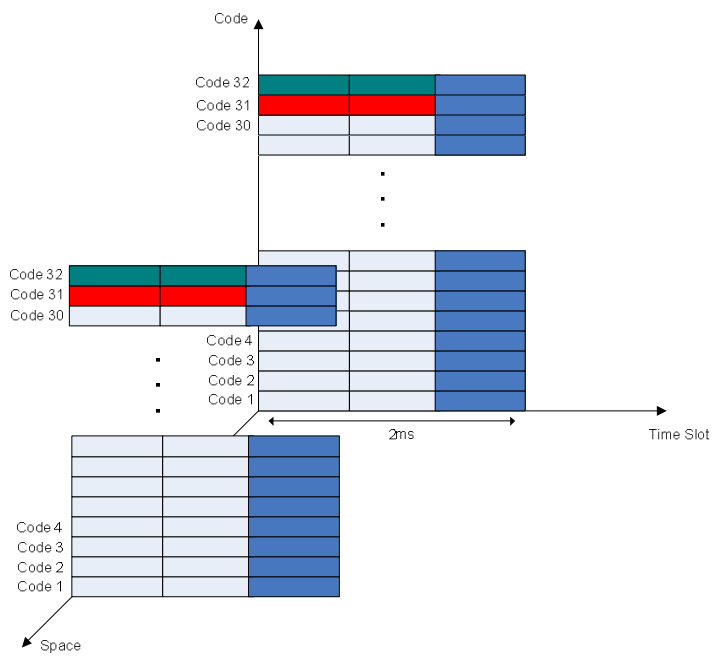

Figure 5 SDMA resource space definition.

In order to exploit spatial diversity the resource allocation mechanism evaluates the Mobile Stations (MSs) AoA on every scheduling period resulting in a spatial diversity list. The packet scheduler is a max C/I scheduler that assigns a priority to each packet according to channel strength. Therefore associated with each packet we have 2-samples, $i$, and $\sigma_{i}$, where $i$ represents the packet order in the maxC/I list (where the users are ordered in terms of decreasing channel strength) and $\sigma_{i}$ is the associated AoA.

Let us define the following parameters

- $N_{c}$ : number of available codes 
- $\quad N_{s}$ : maximum number of spatially separable channels (number of beams supported in the sector)

- $\Delta \theta_{\text {min: }}$ Minimum required AoS for the PHY (Physical) layer to be able to separate two MSs

It is clear that due to the introduction of the spatial dimension, we can increase the number of scheduled packets in one time slot from $N_{c}$ which is the maximum allowable codes with a SISO channel up to a maximum number equal to $N_{s} N_{c}$.

Let us define an array of dimension $N_{s} N_{c}$, where $N_{c}$ is the number of codes and $N_{s}$ the number of spatial columns. The elements of the array will contain packet indices of the packets to be scheduled.

\subsection{H-ARQ}

The type II Automatic repeat request (ARQ) with Chase's combining is adopted as an error control technique. In this hybrid ARQ scheme, all retransmitted packets are combined at the receiver in order to form a more reliable estimate of the transmitted data. The ARQ scheme will be only applied to traffic that is tolerant to delays (web traffic).

\section{NUMERICAL RESULTS}

\subsection{Simulation Scenario}

Tab. 2 summarizes the simulation parameters based on the 4MORE target scenario detailed in [15]. Concerning the traffic models, the full-queue traffic option is characterized by an always-full user transmission buffer; this is the commonly adopted model for evaluating system capacity. For the Near Real Time Video option, the source rate is $2 \mathrm{Mbps}$ and is based on the modelling approach in [16].

Table 2. System-level simulations parameters

\begin{tabular}{|l|l|}
\hline \hline Parameter & Value \\
\hline Environment & Urban \\
\hline Mobile velocity & $3 \mathrm{~km} / \mathrm{h}$ \\
\hline Frame duration & $2 \mathrm{~ms}$ \\
\hline Look-Up Tables & $\begin{array}{l}4 \times 2 \text { MIMO Generated using EESM } \\
\text { methodology. }\end{array}$ \\
\hline Channel Model & 3 GPP 2 \\
\hline Cell type & Sectorized (3 sectors) \\
\hline Cell radius & $300 \mathrm{~m}$ (size of hexagon) \\
\hline Traffic models & $\begin{array}{l}\text {-Full queue } \\
\text {-NRTV 2Mbps }\end{array}$ \\
\hline Users & $\begin{array}{l}-30 \text { per sector Fullqueue } \\
-15 \text { per sector NRTV2Mbps }\end{array}$ \\
\hline $\begin{array}{l}\text { MIMO: Number of BS } \\
\text { Antennas }\end{array}$ & 4 \\
\hline $\begin{array}{l}\text { MIMO: Antenna } \\
\text { separation }\end{array}$ & $0.5 \lambda$ \\
\hline Number codes data & 30 \\
\hline Number slots data & 2 \\
\hline $\begin{array}{l}\text { Max. of beams per } \\
\text { sector }\end{array}$ & 2 \\
\hline
\end{tabular}

\begin{tabular}{|l|l|}
\hline Scheduler type & Max C/I \\
\hline Link Adaptation & BLER $\leq 0.1$
\end{tabular}

Some simplifying assumptions were assumed in the simulations. No spatial beam interference is taken into account for two MSs $M S_{i}$ and $M S_{j}$ separated by angle of separation $\theta_{i, j} \geq \theta_{\text {min }}$, that is 45 degrees.

\subsection{Results}

\subsubsection{Full queue traffic}

Fig. 6 shows the average Over-The-Air (OTA) throughput and average service sector throughput; where the OTA is the total number of bits transmitted over the air-interface, and the service throughput is the number of successfully transmitted bits within the simulation time [12].

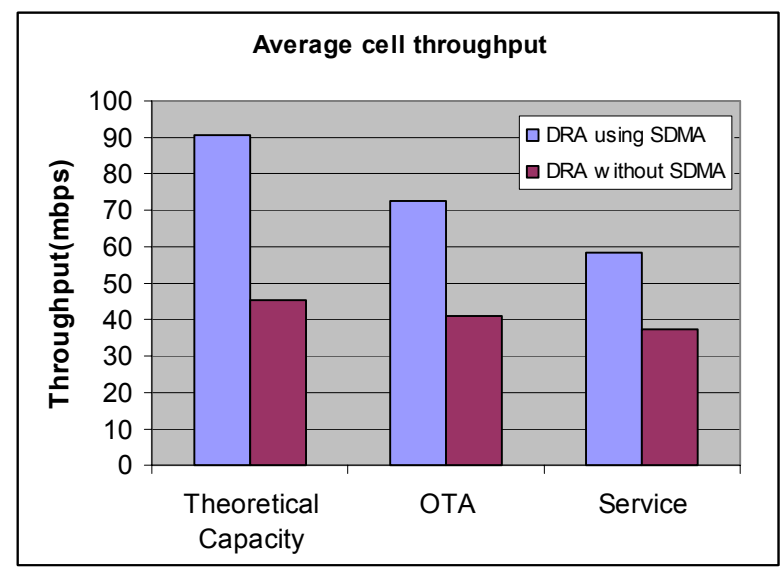

Figure 6 Full queue average sector throughput for DRA with SDMA vs. non-SDMA case

Results have shown that with SDMA, the Over-TheThroughput (OTA) for the full-queue case can reach up to $72 \mathrm{Mbps}$ resulting in a throughout gain of $31 \mathrm{Mbps}$ over non-SDMA DRA case. Lack of diversity gain due to low number of users reduces the simulated SDMA gain. Service Throughput is around $58 \mathrm{Mbps}$ for SDMA and $38 \mathrm{Mbps}$ without SDMA. The discrepancy between service and OTA, although slight, is due to users being serviced at the cell edge which will normally experience poor signal quality.

\subsubsection{NRTV 2 Mbps}

Fig. 7 presents the results of DRA under NRTV traffic for SDMA vs. non-SDMA case. The use of service traffic models means that the effect of SDMA is less pronounced as in the case of the full queue case, especially for NRTV. Furthermore, we notice a $4 \mathrm{Mbps}$ drop in service throughput compared to the OTA. Using traffic models with a pronounced activity factor and large packet sizes will increase the SDMA cell throughput since users with the highest channel 
propagation conditions would be continuously served with the highest MCS option (16 QAM, 3/4 rate encoder) avoiding under utilization of resources.

Fig. 8 presents the percentage of satisfied users. Improved results can be attained by using: greater density of users to improve multi-user diversity gain, and a priority -based scheduler to increase the number of satisfied users. The scheduling policy will affect the trade-off between maximization the cell throughput using SDMA, and keeping the number of satisfied users at an acceptable level.

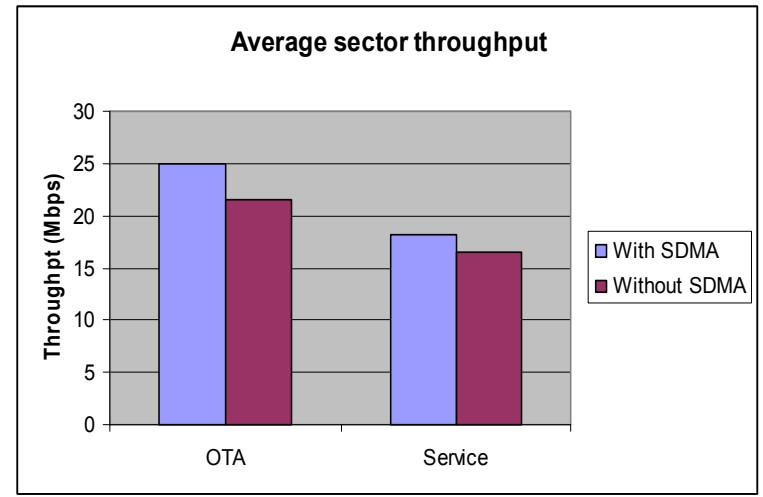

Figure 7. NRTV 2Mbps average sector throughput for SDMA vs. non-SDMA

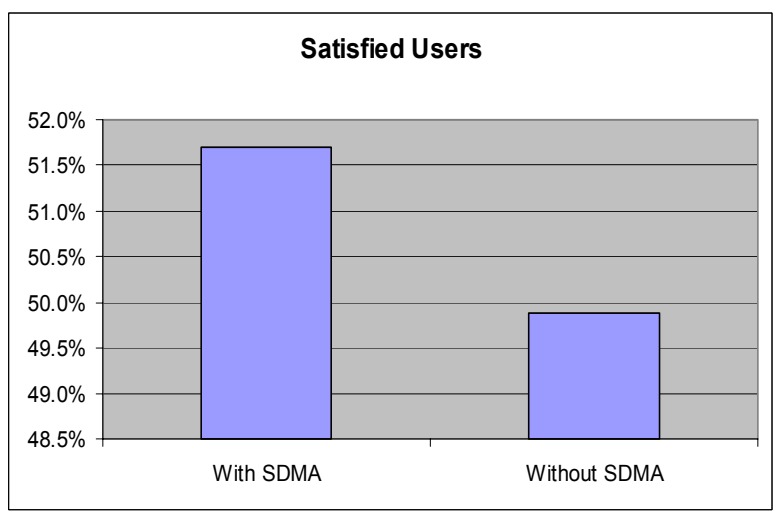

Figure 8. Percentage of satisfied users for NRTV

Obtained results lead to conclude that the performance of SDMA is affected by the likelihood of finding a user pair that has an angle of separation greater than the required separation, in this case 45 degrees. User density will affect the amount of multi-user diversity that can be exploited by the scheduler, a low density will reduce the average MCS utilization option (lowest option corresponds to QPSK with $1 / 2$ rate encoder). A radiation pattern with a lower angular beamwidth will increase the likelihood of finding a user pair within a given coverage area.

We also provide the spectral efficiency of the system for the given deployment strategy and scheduler configuration for SDMA vs. non-SDMA. We define the Spectral Efficiency as the ratio of correctly transmitted bits over the air-interface to the amount of useful bandwidth; units given in $\mathrm{b} / \mathrm{s} / \mathrm{Hz}$. Although there are several variations to this definition, we adopt the $3 \mathrm{GPP}$ definition as presented in Tab. 3 .

Table 3. 4MORE Spectral Efficiency

\begin{tabular}{|c|c|c|}
\hline Symbol & Quantity & Value \\
\hline $\mathrm{W}_{\mathrm{eff}}$ & Effective system bandwidth & $41.7 \mathrm{MHz}$ \\
\hline $\mathrm{L}_{\mathrm{tdd}}$ & TDD Loss & 0,67 \\
\hline $\mathrm{E}_{\text {Band }}$ & $\mathrm{W}_{\text {eff }} * \mathrm{~L}_{\mathrm{tdd}}$ & $27,93 \mathrm{MHz}$ \\
\hline $\mathrm{L}_{\text {guard }}$ & $\begin{array}{l}\text { Loss due to guard times, } \\
\text { UL/DL round trip time etc.. }\end{array}$ & 0,718 \\
\hline$E_{\text {Band }}^{\prime}$ & $\mathrm{E}_{\text {Band } *} \mathrm{~L}_{\text {guard }}$ & $20.06 \mathrm{MHz}$ \\
\hline $\mathrm{L}_{\text {control }}$ & $\begin{array}{l}\text { No. of data codes /Total no. } \\
\text { of codes }\end{array}$ & 0,9375 \\
\hline $\mathrm{U}_{\text {band }}$ & $\begin{array}{l}\text { Useful Band ( } \mathrm{E}_{\text {Band }} * \mathrm{~L}_{\text {guard }} * \\
\left.\mathrm{~L}_{\text {control }}\right)\end{array}$ & $18.80 \mathrm{MHz}$ \\
\hline $\mathrm{S}_{\max }$ & $\begin{array}{l}\text { Achievable Service Sector } \\
\text { Throughput }\end{array}$ & $38.5 \mathrm{Mbps}$ \\
\hline $\mathrm{S}_{\max (\mathrm{SDMA})}$ & $\begin{array}{l}\text { Achievable Service Sector } \\
\text { Throughput (SDMA) }\end{array}$ & $58.5 \mathrm{Mbps}$ \\
\hline $\mathrm{SE}_{\max }$ & $\begin{array}{l}\text { Spectral efficiency (effective } \\
\text { bandwidth })\left(\mathrm{S}_{\max } / \mathrm{E}_{\text {Band }}^{\prime}\right)\end{array}$ & 1.92 \\
\hline $\mathrm{SE}_{\max }$ & $\begin{array}{l}\text { Spectral efficiency (Useful } \\
\text { bandwidth) }\left(\mathrm{S}_{\max } / \mathrm{U}_{\text {band }}\right)\end{array}$ & 2.05 \\
\hline $\mathrm{SE}_{\max (\mathrm{SDMA})}$ & $\begin{array}{l}\text { Spectral efficiency (effective } \\
\text { bandwidth) }\left(\mathrm{S}_{\max } / \mathrm{E}_{\text {Band }}^{\prime}\right)\end{array}$ & 2.92 \\
\hline $\mathrm{SE}_{\max (\mathrm{SDMA})}$ & $\begin{array}{l}\text { Spectral efficiency (Useful } \\
\text { bandwidth) }\left(\mathrm{S}_{\max } / \mathrm{U}_{\text {band }}\right)\end{array}$ & 3.11 \\
\hline
\end{tabular}

\section{CONCLUSION}

A DRA architecture that uses smart antenna to facilitate SDMA was proposed for the 4MORE air-interface. Simulation results have shown that significant capacity gain can be achieved by employing SDMA in the crosslayer design for DRA operating over MC-CDMA MTMR techniques. In this SDMA case study we conclude that user density will affect the amount of multi-user diversity that can be exploited by the DRA. Also, the number of antenna elements will define the theoretical capacity gain, and the radiation pattern will dictate the user SIR distribution in the cell.

In summary, the application of SDMA to DRA led to a 1.5 increase in the spectral efficiency compared to the non-beamforming case.

\section{ACKNOWLEDGEMENT}

The work presented in this paper was partially supported by the IST ACE2 026957 and the FCT CROSSNET POSC/EEA-SRI/57503/2004 projects.

\section{REFERENCES}

1. ITU-R, "Vision, framework and overall objectives of the future development of IMT-2000 and systems beyond IMT-2000", draft new recommendation ITU-R M. [imt-vis] [doc. 8/110], Feb. 2003.

2. IST MATRICE, http://www.ist-MATRICE.org/, IST- 200132620 
3. Liang $\mathrm{Xu}$, Xuemin Shen, and Jon W. Mark, Dynamic bandwidth allocation with fair scheduling for WCDMA systems, IEEE Wireless Communications, vol. 9, no. 2, April 2002, pp. 2632

4. A. Stamoulis, N. D. Sidiropoulos, G. B. Giannackis, Time-varying fair queueing scheduling for multicode CDMA based on dynamic programming, IEEE transactions on Wireless Communications, vol. 3, Issue 2, March 2004, pp. $512-523$

5. L. Xu, X Shen, J. W. Mark, Aggressive CodeDivision Generalized Processor Sharing for QoS guarantee in Multimedia CDMA Cellular Networks, Proc. IEEE Globecom02 ieeeplore.ieee.org

6. IST 4MORE, http://www.ist-4more.org/, IST- 2002 507039

7. Godara, L., "Application of Antenna Arrays to Mobile Communications, Part II: Beamforming and Direction-of-Arrival Considerations", Proceedings of the IEEE, Aug. 1997, vol. 85, No 8, pp.11951245

8. Hara, Y. "Time Slot Assignment Algorithm for SDMA/TDMA System Based on Estimated SINR", IEICI Trans. Commun., Vol E84-B, No. 2, Feb 2001

9. C. Hoymann, H. Meng, "Influence of SDMASpecific MAC Scheduling on the Performance of IEEE 802.16 Networks", $12^{\text {th }}$ Europeean Wireless Conference, Athens, 2-5 April 2006

10. 3GPP, TR25.996 "Spatial channel model for multiple Input Multiple Output (MIMO) simulations," v6.1.0, 2003-09

11. D. T. Phan Huy, V. Monteiro, A. Gameiro, J. Rodriguez, "System level performance evaluation of MATRICE air interface”, Proceedings of ISTSummit 2004

12. 3GPP, TR25.848 " Physical layer aspects of UTRA High Speed Downlink Packet Access ," v4.0.0, 2001-03

13. J. Rodriguez, V. Monteiro, R. Legouable, n. Ibrahim, M. Shateri, "4MORE System Level Performance", VI International Telecommunications Symposium (ITS2006), September 3-6, 2006, Fortaleza-CE, Brazil

14. Ericsson, "Effective SNR mapping for modelling frame error rates in multiple-state channels," 3GPP2-C30-20030429-010

15. IST-4MORE, "Intermediate version evaluation platform including a description of simulation library," Deliverable D2.2a, 4MORE IST-2002 507039

16. 3GPP, TR25.892, "Feasibility Study for Orthogonal Frequency Division Multiplexing (OFDM) for UTRAN enhancement," v6.0.0, 200406. 